\title{
PICARD VALUES AND DERIVATIVES OF MEROMORPHIC FUNCTIONS*
}

\author{
YAN XU
}

\begin{abstract}
Let $f$ be a transcendental meromorphic function and $R$ be a rational function, $R \not \equiv 0$, and let $k$ be a positive integer. In this paper, we obtain some results concerning the zeros of $f^{(k)}-R$, which generalize and improve related results of Wang-Fang and Bergweiler-Pang.
\end{abstract}

\section{Introduction}

In 1998, Wang and Fang [11] proved the following results.

THEOREM A. Let $f$ be a transcendental meromorphic function and $k \in \mathbf{N}$. If $f$ has only zeros of multiplicity at least $k+1$ and poles of multiplicity at least 2, then, for each $c \in \mathbf{C} \backslash\{0\}, f^{(k)}-c$ has infinitely many zeros.

THEOREM B. Let $f$ be a transcendental meromorphic function and $k \in \mathbf{N}$. If $f$ has only zeros of multiplicity at least 3, then, for each $c \in \mathbf{C} \backslash\{0\}, f^{(k)}-c$ has infinitely many zeros.

A natural problem arises: What can we say if the constant $c$ in the above results is replaced by a rational function $R(z) \not \equiv 0$ ? (see [1]).

In 2000, Fang [3] considered the fixed points of $f^{\prime}$ and obtained

THEOREM C. Let $f$ be a transcendental meromorphic function. If $f$ has only multiple zeros and poles, then $f^{\prime}-z$ has infinitely many zeros.

Recently, Bergweiler and Pang [2] proved the following result, which is a significant improvement of Theorem $\mathrm{C}$.

2000 Mathematics Subject Classification: 30D35, 30D45.

Key words and phrases: transcendental meromorphic function, normal family, Julia exceptional function.

* The author was partially supported by NSF of China (Grant 10471065) and NSF of Education Department of Jiangsu Province (Grant 03KJB110058).

Received March 24, 2004. 
THEOREM D. Let $f$ be a transcendental meromorphic function, and let $R$ be a rational function, $R \neq \equiv$. Suppose that all zeros and poles of $f$ are multiple, except possibly finite many, then $f^{\prime}-R$ has infinitely many zeros.

In this paper, we borrow the idea of Bergweiler and Pang [2] and prove the following results with a simpler proof.

THEOREM 1. Let $f$ be a transcendental meromorphic function, and let $R$ be a rational function, $R \neq \equiv, k \in \mathbf{N}$. Suppose that all zeros of $f$ have multiplicity at least $k+1$ and all poles of $f$ have multiplicity at least 2 , except possibly finite many. Then $f^{(k)}-R$ has infinitely many zeros.

THEOREM 2. Let $f$ be a transcendental meromorphic function, and let $R$ be a rational function, $R \neq \equiv 0, k \in \mathbf{N}$. Suppose that all zeros of $f$ have multiplicity at least $k+2$, except possibly finite many. Then $f^{(k)}-R$ has infinitely many zeros.

It seems reasonable to conjecture that the conclusion of Theorem 1 or Theorem 2 still holds under a considerably weaker condition that the zeros of $f$ have multiplicity at least 3 . In this direction, we have the following result.

THEOREM 3. Let $f$ be a transcendental meromorphic function, $k \in \mathbf{N}$, and let $P$ be a polynomial, $P \neq \equiv$. If all zeros of $f$ have multiplicity at least 3 , except possibly finite many, then $f^{(k)}-P$ has infinitely many zeros.

We shall use some standard notations and results from Nevanlinna theory (see $[4,9,12])$.

\section{Some Lemmas}

Now we recall some definitions. If there exists a curve $\Gamma \subset \mathbf{C}$ tending to $\infty$ such that $f(z) \rightarrow a$ as $z \rightarrow \infty, z \in \Gamma$, we call that $a$ is an asymptotic value of $f$. A meromorphic function $f$ is called a Julia exceptional function if $f^{\#}(z)=$ $O(1 /|z|)$ as $z \rightarrow \infty$. Here, as usual, $f^{\#}(z)=\left|f^{\prime}(z)\right| /\left(1+|f(z)|^{2}\right)$ is the spherical derivative of $f$. It follows easily from the Ahlfors-Shimizu form of the Nevanlinna characteristic function that if $f$ is a Julia exceptional function, then $T(r, f)=O\left((\log r)^{2}\right)$ as $r \rightarrow \infty$. The following result concerning functions satisfying this growth condition is due to Hayman $[5,6]$.

LEMMA 1. Let $f$ be an entire function satisfying

$$
\log M(r, f)=O\left((\log r)^{2}\right)
$$

as $r \rightarrow \infty$. Then $\log \left|f\left(r e^{i \theta}\right)\right| \sim \log M(r, f)$ as $r \rightarrow \infty$ for almost every $\theta \in[0,2 \pi]$.

The following result is due to Lehto and Virtanen [7]. 
Lemma 2. A transcendental Julia exceptional function does not have an asymptotic value.

LEMma 3. Let $f$ be a transcendental meromorphic function and $k \in \mathbf{N}$, and let $R$ be a rational function satisfying $R(z) \sim c z^{d}$ as $z \rightarrow \infty$, with $c \in \mathbf{C} \backslash\{0\}$ and $d \in \mathbf{Z}$. Suppose that $f^{(k)}-R$ has only finitely many zeros and that $T(r, f)=$ $O\left((\log r)^{2}\right)$ as $r \rightarrow \infty$. Set $g:=f(z) / z^{d+k}$. Then $g$ has an asymptotic value.

Proof. Since $f^{(k)}-R$ has only finitely many zeros, there exists a polynomial $P \not \equiv 0$ such that $h=P /\left(f^{(k)}-R\right)$ is entire. By standard results in Nevanlinna theory, we have

$$
\log M(r, h) \leq 3 T(2 r, h) \leq 3 T\left(2 r, f^{(k)}\right)+O(\log r)
$$

and

$$
T\left(2 r, f^{(k)}\right) \leq(k+1) T(2 r, f)+O(\log r)
$$

as $r \rightarrow \infty$. Thus $\log M(r, h)=O\left((\log r)^{2}\right)$. By Lemma 1, there exists $\theta \in[0,2 \pi]$ such that $\left|h\left(r e^{i \theta}\right)\right| r^{-m} \rightarrow \infty(r \rightarrow \infty)$ for any $m \in \mathbf{Z}$. Let $m=\operatorname{deg}(P)+2+|d|$. It follows that

$$
\left|f^{(k)}\left(r e^{i \theta}\right)-R\left(r e^{i \theta}\right)\right|=\left|\frac{P\left(r e^{i \theta}\right)}{h\left(r e^{i \theta}\right)}\right| \leq \frac{1}{r^{2+|d|}}
$$

for sufficiently large $r$, say $r \geq r_{0}$. Hence

$$
\int_{r_{0}}^{r}\left(f^{(k)}\left(t e^{i \theta}\right)-R\left(t e^{i \theta}\right)\right) d t
$$

tends to a finite limit as $r \rightarrow \infty$. We consider three cases.

CASE 1. $d>-1$. We have

$$
\lim _{r \rightarrow \infty} \frac{f^{(k-1)}\left(r e^{i \theta}\right)}{\frac{c}{d+1}\left(r e^{i \theta}\right)^{d+1}}=1 .
$$

Hence

$$
\begin{aligned}
\lim _{z \rightarrow \infty} g(z) & =\lim _{z \rightarrow \infty} \frac{f(z)}{z^{d+k}}=\lim _{z \rightarrow \infty} \frac{f^{\prime}(z)}{(d+k) z^{d+k-1}}=\cdots \\
& =\lim _{z \rightarrow \infty} \frac{f^{(k-1)}(z)}{(d+k)(d+k-1) \cdots(d+2) z^{d+1}}=\frac{c}{(d+1)(d+2) \cdots(d+k)} .
\end{aligned}
$$

CASE 2. $d<-1$. We have

$$
f^{(k-1)}(z)=a+\frac{c}{d+1} z^{d+1}+O\left(|z|^{d}\right)
$$


for some $a \in \mathbf{C}$ as $z \rightarrow \infty$. For $k=1$, we have either $\lim _{z \rightarrow \infty} g(z)=\infty$ or $\lim _{z \rightarrow \infty} g(z)=c /(d+1)$ (see [2]). For $k \geq 2$, by an elemental calculation, we have that

(a) if $-k \leq d<-1$, then $\lim _{z \rightarrow \infty} g(z)=\infty$;

(b) if $d<-k$, then either $\lim _{z \rightarrow \infty} g(z)=\infty$ or

$$
\lim _{z \rightarrow \infty} g(z)=\frac{c}{(d+1)(d+2) \cdots(d+k)} .
$$

CASE 3. $d=-1$. We have

$$
\lim _{z \rightarrow \infty} \frac{f^{(k-1)}(z)}{c \log |z|}=1
$$

Then

$$
\lim _{z \rightarrow \infty} g(z)=\lim _{z \rightarrow \infty} \frac{f(z)}{z^{k-1}}=\lim _{z \rightarrow \infty} \frac{f^{\prime}(z)}{(k-1) z^{k-2}}=\cdots=\lim _{z \rightarrow \infty} \frac{f^{(k-1)}(z)}{(k-1) !}=\infty .
$$

This completes the proof of Lemma 3 .

LEMma 4 ([11]). Let $f$ be a meromorphic function of finite order in the plane, $b$ nonzero complex numbers, and $k$ a positive integer. If all zeros of $f$ are of order at least $k+1$ and all poles of $f$ are multiple, and $f^{(k)}(z) \neq b$, then $f(z)$ is a constant.

Lemma 5 ([11]). Let $f$ be a meromorphic function of finite order in the plane, $b$ nonzero complex numbers, and $k$ a positive integer. If all zeros of $f$ are of order at least $k+2$ and $f^{(k)}(z) \neq b$, then $f(z)$ is a constant.

The next is one up-to-date version of Zalcaman's lemma due to Pang and Zalcman [8].

Lemma 6. Let $k$ be a positive integer and let $\mathscr{F}$ be a family of functions meromorphic in a domain $D$, such that each function $f \in \mathscr{F}$ has only zeros of multiplicity at least $k$, and suppose that there exists $A \geq 1$ such that $\left|f^{(k)}(z)\right| \leq A$ whenever $f(z)=0$. If $\mathscr{F}$ is not normal at $z_{0} \in D$, then, for each $0 \leq \alpha \leq k$, there exist a sequence of points $z_{n} \in D, z_{n} \rightarrow z_{0}$, a sequence of positive numbers $\rho_{n} \rightarrow 0$, and a sequence of functions $f_{n} \in \mathscr{F}$ such that

$$
g_{n}(\zeta)=\frac{f_{n}\left(z_{n}+\rho_{n} \zeta\right)}{\rho_{n}^{\alpha}} \rightarrow g(\zeta)
$$

locally uniformly with respect to the spherical metric, where $g$ is a nonconstant meromorphic function on $C$, all of whose zeros have multiplicity at least $k$, such that $g^{\#}(\zeta) \leq g^{\#}(0)=k A+1$. Moreover, $g$ has order at most 2 . 


\section{Proof of Theorems}

Proof of Theorem 1. Suppose that $f^{(k)}-R$ has finitely many zeros. We assume that $R(z) \sim c z^{d}$ as $z \rightarrow \infty$, with $c \in \mathbf{C} \backslash\{0\}$ and $d \in \mathbf{Z}$. Define

$$
g(z):=f(z) / z^{d+k} \text {. }
$$

If $g$ is a Julia exceptional function, then $T(r, g)=O\left((\log r)^{2}\right)$ and hence $T(r, f)=$ $O\left((\log r)^{2}\right)$ as $r \rightarrow \infty$. Thus, by Lemma 3, $g$ has an asymptotic value. But $g$ has not an asymptotic value by Lemma 2 , a contradiction.

Thus $g$ is not a Julia exceptional function, and then there exists a sequence $\left(a_{n}\right)$ in $\mathbf{C}$ such that $a_{n} \rightarrow \infty$ and $a_{n} g^{\#}\left(a_{n}\right) \rightarrow \infty$ as $n \rightarrow \infty$. Let $D=$ $\{z \in \mathbf{C}:|z-1|<1 / 2\}$, and set

$$
\mathscr{F}=\left\{g_{n}(z):=g\left(a_{n} z\right) z^{d+k}=\frac{f\left(a_{n} z\right)}{a_{n}^{d+k}}, n=1,2,3 \ldots, z \in D\right\} .
$$

Since

$$
g_{n}^{\#}(1)=\frac{\left|a_{n} g^{\prime}\left(a_{n}\right)+(d+k) g\left(a_{n}\right)\right|}{1+\left|g\left(a_{n}\right)\right|^{2}} \geq\left|a_{n}\right| g^{\#}\left(a_{n}\right)-\frac{|d+k|}{2} \rightarrow \infty
$$

as $n \rightarrow \infty$, we know that $\mathscr{F}$ is not normal at 1 . Obviously, for sufficiently large $n$, all zeros of $g_{n}$ in $D$ have multiplicity at least $k+1$ and all poles of $g_{n}$ are multiple. Then by Lemma 6 , we can find sequences $\left(n_{j}\right),\left(z_{j}\right)$ and $\left(\rho_{j}\right)$ satisfying $n_{j} \in \mathbf{N}, n_{j} \rightarrow \infty, z_{j} \in D, z_{j} \rightarrow 1, \rho_{j}>0$ and $\rho_{j} \rightarrow 0$ such that

$$
G_{n_{j}}(\zeta)=\frac{g_{n_{j}}\left(z_{j}+\rho_{j} \zeta\right)}{\rho_{j}^{k}}=\frac{f\left(a_{n_{j}}\left(z_{j}+\rho_{j} \zeta\right)\right)}{\rho_{j}^{k} a_{n_{j}}^{d+k}} \rightarrow G(\zeta)
$$

locally uniformly with respect to the spherical metric, where $G(\zeta)$ is a nonconstant meromorphic function in $\mathbf{C}$, all of whose zeros have multiplicity at least $k+1$. In particular, $G$ is of order at most 2. By Hurwitz's theorem, $G$ has only multiple poles.

Since $R(z) \sim c z^{d}$ as $z \rightarrow \infty$, we have

$$
G_{n_{j}}^{(k)}(\zeta)-\frac{R\left(a_{n_{j}}\left(z_{j}+\rho_{j} \zeta\right)\right)}{a_{n_{j}}^{d}} \rightarrow G^{(k)}(\zeta)-c
$$

and (for $j$ sufficiently large)

$$
G_{n_{j}}^{(k)}(\zeta)-\frac{R\left(a_{n_{j}}\left(z_{j}+\rho_{j} \zeta\right)\right)}{a_{n_{j}}^{d}}=\frac{f^{(k)}\left(a_{n_{j}}\left(z_{j}+\rho_{j} \zeta\right)\right)-R\left(a_{n_{j}}\left(z_{j}+\rho_{j} \zeta\right)\right)}{a_{n_{j}}^{d}} \neq 0 .
$$

It follows from Hurwitz's theorem that either $G^{(k)} \neq c$ or $G^{(k)} \equiv c$ on C. But $G^{(k)} \not \equiv c$ since all zeros of $G$ have multiplicity at least $k+1$. Thus $G^{(k)} \neq c$. By Lemma 4, $G(\zeta)$ must be a constant, a contradiction. This completes the proof of Theorem 1. 
Proof of Theorem 2. Theorem 2 can be proved by using Lemma 5 and the same argument as in Theorem 1. We here omit the details.

Proof of Theorem 3. For $k=1$, the conclusion comes from Theorem 2. Now we assume $k \geq 2$. Let $P(z)=a_{n} z^{n}+a_{n-1} z^{n-1}+\cdots+a_{0} \quad\left(a_{i} \in \mathbf{C}, i=\right.$ $\left.0,1, \ldots, n, a_{n} \neq 0\right)$. Suppose that $f^{(k)}-P$ has finitely many zeros. Then

$$
N\left(r, \frac{1}{f^{(k)}-P}\right)=S(r, f) .
$$

By the logarithmic derivative theorem, we have

$$
\begin{aligned}
m\left(r, \frac{1}{f}\right) & =m\left(r, \frac{1}{f^{(k)}-P}\right) \\
& =m\left(r, \frac{f^{(k+n)}}{f} \frac{1}{f^{(k+n)}}\right)+m\left(r, \frac{\left(f^{(k)}-P\right)^{(n)}}{f^{(k)}-P} \frac{1}{\left(f^{(k)}-P\right)^{(n)}}\right) \\
& \leq m\left(r, \frac{1}{f^{(k+n)}}\right)+m\left(r, \frac{1}{f^{(k+n)}-a_{n} n !}\right)+S(r, f) \\
& =m\left(r, \frac{1}{f^{(k+n)}}+\frac{1}{f^{(k+n)}-a_{n} n !}\right)+S(r, f) \\
& \leq m\left(r, \frac{1}{f^{(k+n+1)}}\right)+S(r, f) \\
& \leq T\left(r, f^{(k+n+1)}\right)-N\left(r, \frac{1}{f^{(k+n+1)}}\right)+S(r, f) . \\
& \leq T\left(r, f^{(k)}\right)+(n+1) \bar{N}(r, f)-N\left(r, \frac{1}{f^{(k+n+1)}}\right)+S(r, f) .
\end{aligned}
$$

Thus

$T(r, f) \leq(n+1) \bar{N}(r, f)+N\left(r, \frac{1}{f}\right)+N\left(r, \frac{1}{f^{(k)}-P}\right)-N\left(r, \frac{1}{f^{(k+n+1)}}\right)+S(r, f)$.

Using a inequality of Wang [10] and noting that $k \geq 2$, then, for every $\varepsilon>0$, we have

$$
\begin{aligned}
(n+1) \bar{N}(r, f)+N\left(r, \frac{1}{f}\right) & \leq(k+n-1) \bar{N}(r, f)+N\left(r, \frac{1}{f}\right) \\
& \leq 2 \bar{N}\left(r, \frac{1}{f}\right)+N\left(r, \frac{1}{f^{(k+n+1)}}\right)+\varepsilon T(r, f)+S(r, f) .
\end{aligned}
$$

Combining (2) and (3), we obtain 


$$
T(r, f) \leq 2 \bar{N}\left(r, \frac{1}{f}\right)+N\left(r, \frac{1}{f^{(k)}-P}\right)+\varepsilon T(r, f)+S(r, f) .
$$

Recalling that the zeros of $f$ are of order $\geq 3$ and setting $\varepsilon=\frac{1}{6}$, from (1) and (4), we get

$$
T(r, f) \leq 6 N\left(r, \frac{1}{f^{(k)}-P}\right)+S(r, f)=S(r, f),
$$

which contradicts the fact that $f$ is a transcendental meromorphic function. Theorem 3 is proved.

Acknowledgement. We wish to thank Professor H. H. Chen for helpful suggestions.

\section{REFERENCES}

[1] W. BergweIler, 'On the product of a meromorphic function and its derivatives', Bull. Hong Kong Math. Soc. 1 (1997), 97-101.

[2] W. Bergweiler and X. C. PANG, 'On the derivatives of meromorphic functions with multiple zeros', J. Math. Anal. Appl. 278 (2003), 285-292.

[ 3 ] M. L. FANG, 'A note on a problem of Hayman', Analysis 20 (2000), 45-49.

[4] W. K. Hayman, Meromorphic Functions (Clarendon Press, Oxford, 1964).

[5] W. K. Hayman, 'Slowly growing integral and subharmonic functions', Comment. Math. Helv. 34 (1960), 75-84.

[6] W. K. Hayman, Subharmonic functions 2 (Academic Press, London, New York, San Francisco, 1989).

[ 7 ] O. Lehto and K. L. Virtanen, 'On the behavior of meromorphic functions in the neighborhood of an isolated singularity’, Ann. Acad. Sci. Fenn., Ser. A, 240 (1957).

[ 8 ] X. C. Pang And L. Zalcman, 'Normal families and shared values', Bull. London Math. Soc. 32 (2000), 325-331.

[9] J. SchiFf, Normal Families (Springer-Verlag, New York/Berlin, 1993).

[10] Y. F. WANG, 'On Mues conjecture and Picard values', Sci. China (1) 36 (1993), 28-35.

[11] Y. F. WANG AND M. L. FANG, 'Picard values and normal families of meromorphic functions with multiple zeros', Acta Math. Sinica (N.S.) (1) 14 (1998), 17-26.

[12] L. YANG, Value Distribution Theory (Springer-Verlag \& Science Press, Berlin, 1993).

School of Mathematics \& Computer SCIENCE

NANJING NORMAL UNIVERSITY

NANJING 210097, P.R. China

E-mail: xuyan@njnu.edu.cn 